\title{
Flow control of weakly non-parallel flows: application to trailing vortices
}

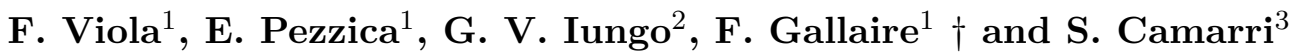 \\ ${ }^{1}$ Laboratory of Fluid Mechanics and Instabilities, École Polytechnique Fédérale de Lausanne, \\ Lausanne, Switzerland \\ ${ }^{2}$ Wind Fluids and Experiments (WindFluX) Lab, University of Texas Dallas, Texas, USA. \\ ${ }^{3}$ Department of Civil and Industrial Engineering, University of Pisa, Pisa 56122, Italy
}

(Received ?; revised ?; accepted ?. - To be entered by editorial office)

A general formulation is proposed to control the integral amplification factor of harmonic disturbances in weakly non-parallel amplifier flows. The sensitivity of the local spatial stability spectrum to a base-flow modification is first determined, generalizing the results of Bottaro et al. (2003). This result is then used to evaluate the sensitivity of the overall spatial growth to a modification of the inlet flow condition. This formalism is applied to a non-parallel Batchelor vortex which is a well-known model for trailing vortices generated by a lifting wing. The resulting sensitivity map indicates the optimal modification of the inlet flow condition enabling to stabilize the helical modes. It is shown that the control, formulated using a single linearization of the flow dynamics carried out on the uncontrolled configuration, successfully reduces the total spatial amplification of all convectively unstable disturbances.

\section{Key words:}

\section{Introduction}

In the framework of linear stability analysis, open flows showing self-sustained oscillations, such as spiral vortex-breakdown (Ruith et al. 2003; Qadri et al. 2013), are globally unstable and are called oscillators. In contrast, stable flows which exhibit a strong response to external disturbances are named amplifiers. Trailing vortices generated by a lifting wing are a classical example of amplifier flows in aeronautics (Spalart 1998) and are commonly modeled by using the self-similar solution proposed by Batchelor (1964). In its parallel approximation the stability properties of the Batchelor vortex have been well characterized in the literature. For instance, Lessen et al. (1974) and Mayer \& Powell (1992) found that inviscid helical modes are temporally unstable and higher wavenumber modes are destabilized when the swirl increases (Duck \& Foster 1980). These helical modes reach their maximum growth rate at a certain swirl number (Leibovich \& Stewartson 1983) before getting stable when the azimuthal velocity of the vortex further increases. The mechanism underlying this destabilization is a generalized centrifugal instability unravelled by Ludwieg (1962), Leibovich \& Stewartson (1983) and Eckhoff (1984). On the other hand, viscous modes have been investigated numerically and asymptotically by Khorrami (1991); Heaton (2007); Fabre \& Jacquin (2004); Fabre et al. (2006), whereas Delbende et al. (1998); Olendraru et al. (1999); Olendraru \& Sellier

$\dagger$ Email address for correspondence: francois.gallaire@epfl.ch 
(2002) carried out a spatio-temporal analysis showing that in the case of strong advection and moderate wake deficit (approximately less than $80 \%$ of the external flow) the flow is convectively unstable. More recently, Heaton et al. (2009) carried out a global stability analysis of a non-parallel Batchelor vortex with an inflow wake deficit of $90 \%$ that is locally absolutely unstable close to the inlet and globally unstable. In the framework of amplifier flows, Viola et al. (2016) investigated the response to harmonic inlet and body forcing of a globally stable non-parallel vortex by a using local WKB approach and a global resolvent analysis.

These studies suggest that the stability properties of swirling flows depend in a complex way on multiple factors, such as the advection rate, the swirl number, the presence of localized forces, turbulence level, fluid properties, domain geometry and boundary conditions. Thus, a modification of one of these factors yields a variation of the linearized operators of the stability analysis which turns into a modification of the eigenvalues in the stability spectrum. The dependence of the stability properties on one or more of these factors is commonly called sensitivity. Chomaz (2005) explained that the sensitivity may become large when the linear stability operator is non-normal, which is usually the case in hydrodynamics. For this reason several studies have been devoted over the years to sensitivity analyses. Giannetti \& Luchini (2007) defined the so-called structural sensitivity, which is the sensitivity to a spatially localized feedback and showed that the combined analysis of the global mode and its adjoint mode allows the identification of the wavemaker of the stability, i.e. the core region where the self-excited instability mechanism takes place. Bottaro et al. (2003) were the first to study the sensitivity to a base-flow modification and they identified the regions of the parallel Couette flow where a base flow modification has the most significative effect on temporal stability. Such base-flow variations can be the result of a passive control acting on the flow. Using a variational technique, Marquet et al. (2008) derived a general expression of the base-flow sensitivity valid for oscillator flows.

In the case of amplifiers, the flow response to an external forcing is given by the resolvent analysis (Chomaz 2005) and its sensitivity with respect to a base-flow modification was derived analytically by Brandt et al. (2011). They focused on the variation of the resolvent norm in the case of the Blasius boundary layer subjected to a harmonic body force where both component-wise and convective non-normalities are present. This analysis was applied by Boujo \& Gallaire (2015) to the backward-facing step flow and extended to the case of inlet forcing and time-stochastic perturbation. However, when the base flow evolves slowly in the advection direction these global approaches overlook the physical amplification mechanism, which is the convective growth of perturbation that can be related to the local stability properties of the flow. Indeed, by using both a local WKB asymptotic analysis (Crighton \& Gaster 1976) and a global resolvent approach (Åkervik et al. 2008) for a non-parallel Batchelor vortex, Viola et al. (2016) showed that the linear global amplification of perturbations in amplifier flows is well captured by the spatial stability branches. This raises the question if it is possible to determine a systematic way to control the spatial amplification of a given mode, i.e. its global amplification, resulting from the integration of the local spatial growth along the streamwise direction.

The objective of this paper is to provide a general framework so as to solve the mentioned control problem. The method proposed here generalizes to the case of weakly non-parallel amplifier flows the adjoint-based strategies usually employed for controlling oscillators (see Camarri (2015) for a review). Noise amplifiers comprise many fundamental flows as for instance boundary layers, convectively unstable wakes, trailing vortices, thus the proposed method is general and has many direct engineering applications. In this paper we present only a particular prototypical application, which consists in con- 
a)

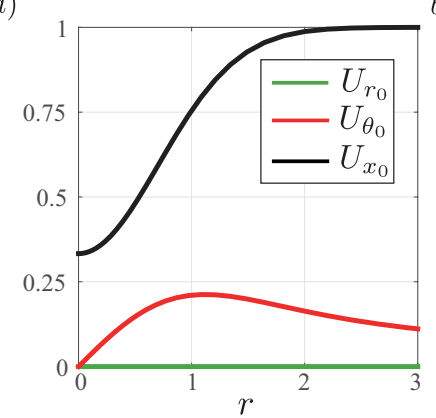

b)

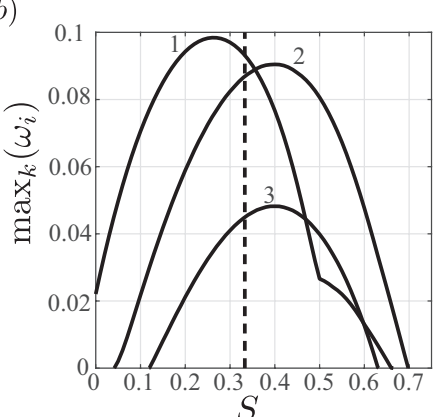

c)

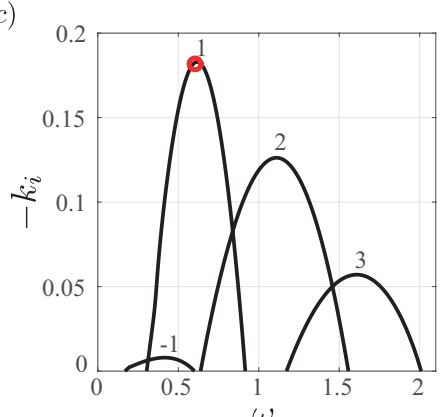

FiguRE 1. (a) Velocity components of the parallel Batchelor vortex with wake and swirl parameters equal to $\alpha=0.667$ and $S=0.333$, respectively. (b) Temporal growth rate maximized over the streamwise wavenumber, $\max _{k}\left(\omega_{i}\right)$, for increasing swirl number at $R e=100, \alpha=0.667$ and azimuthal modes $m=1,2,3$. The vertical line depicts the swirl number used in the paper ( $S=0.333$ ). (c) Local spatial stability properties of the flow, where the spatial growth rate, $-k_{i}$ of the unstable helical perturbations $m \in\{-1,1,2,3\}$ is reported as a function of the frequency $\omega$. The red circle refers to the maximum $-k_{i}$, which corresponds to $m=1$ and $\omega=0.6$.

trolling the spatial instability of the non-parallel Batchelor vortex using a perturbation of the velocity profile generating the vortex.

The particular selected test case, which is aimed at showing an example of flow control, is loosely applicable to disturbances suppression in trailing vortices (Spalart 1998) and in wind turbine wakes (Iungo et al. 2013). To this purpose we need first to address in section 2 the sensitivity of the local spatial stability problem in the parallel flow framework. The non-parallel Batchelor vortex flow and its local stability properties are presented in section 3. Successively, on the basis of the previous result, the sensitivity of the integral amplification factor of a single helical disturbance to a modification of the inflow vortex velocity is derived in section 4 . Furthermore, a strategy to design the passive inlet control to stabilize all helical modes amplified over the vortex flow is proposed in section 5 . Lastly, conclusions and possible applications are discussed in section 6 .

\section{Sensitivity of the local spatial stability spectrum}

\subsection{Parallel flow and spatial stability analysis}

Although the formalism used here applies to all kind of convectively unstable shear flows, we consider now a Batchelor vortex profile with dimensionless velocity components

$$
U_{r}=0, \quad U_{\theta}=S\left(1-e^{-r^{2}}\right) / r, \quad U_{x}=1-\alpha e^{-r^{2}},
$$

which are shown in Figure 1(a). Here, $r, \theta$ and $x$ are the cylindrical coordinates and $\alpha$, $S$ are the wake and swirl parameters and $R e$ designates the Reynolds number, which is based on the vortex core radius and on the free-stream axial velocity. The behaviour of infinitesimal three-dimensional disturbances, $\mathbf{q}(x, r, \theta ; t)=(\mathbf{u}, p)$, superimposed on top of the base-flow is described by the linearized Navier-Stokes equations

$$
\frac{\partial \mathbf{u}}{\partial t}+\mathbf{U} \cdot \nabla \mathbf{u}+\mathbf{u} \cdot \nabla \mathbf{U}=-\nabla p+\frac{1}{R e} \Delta \mathbf{u}, \quad \nabla \cdot \mathbf{u}=0
$$

where $\mathbf{u}$ and $p$ are the disturbance velocity and pressure. In the case of parallel axisymmetric base-flow, these perturbations can be decomposed in the standard form

$$
\mathbf{q}(x, r, \theta ; t)=\hat{\mathbf{q}}(r) e^{i(k x+m \theta-\omega t)},
$$


where $m$ is the azimuthal wavenumber and $\hat{\mathbf{q}}(r)=(\hat{\mathbf{u}}, \hat{p})$.

The wake parameter and Reynolds number are here set to $\alpha=0.667$ and $R e=100$ respectively, and the swirl parameter, $S$, is chosen in such a way to ensure the simultaneous amplification of different helical modes in the swirling wake. By recalling that in temporal analysis framework $k$ is real and $\omega$ is taken complex, figure 1(b) displays the temporal growth rate, $\omega_{i}$, maximized over the streamwise wavenumber, $k$, for $m=1,2,3$ and increasing swirl parameter. It shows strong competition between the first and second helical mode at $S=0.333$, which is further considered in this study. Such a competition is representative of several swirling jet/wake experiments (Gallaire \& Chomaz 2003; Iungo et al. 2013). The considered test-case vortex was shown to be convectively unstable by Viola et al. (2016), calling for a spatial stability analysis, where the frequency $\omega$ is real and wavenumber $k$ complex. The real part of $k, k_{r}$, corresponds to the streamwise wavenumber of the traveling perturbation and the imaginary part of $k$, in particular $-k_{i}$, is the spatial amplification rate. The equations governing the linearized dynamics of $\hat{\mathbf{q}}$, obtained by substituting the normal mode expansion (2.3) in equations (2.2), are reported in appendix A. They can be recast in a quadratic eigenvalue problem of the following type

$$
k^{2} \mathcal{C}_{2}(\mathbf{U}, \omega) \hat{\mathbf{q}}+k \mathcal{C}_{1}(\mathbf{U}, \omega) \hat{\mathbf{q}}+\mathcal{C}_{0}(\mathbf{U}, \omega) \hat{\mathbf{q}}=0,
$$

where the linear operators $\mathcal{C}_{0}, \mathcal{C}_{1}, \mathcal{C}_{2}$, together with boundary conditions are also reported in the appendix A. The discretization is ensured through a Chebyshev spectral collocation method including an algebraic mapping of the domain, as detailed in Viola et al. (2014), where the influence of the radial extension of the domain is discussed in appendix B. In Figure 1(c) the spatial growth rates are shown as a function of the frequency $\omega$, and the integer numbers at the curves' peaks depict their azimuthal wavenumber mode, $m$. In particular four helical modes are seen to be amplified in space and $m=1$ at a frequency approximately equal to 0.6 is the dominant mode. For this particular case, ( $m=1, \omega=0.6)$, the eigenvalue spectrum that is obtained by solving numerically equations (2.4), is reported in Figure 2(a): unstable perturbations propagating downstream are characterized by a positive phase velocity, $\omega / k_{r}$, and a negative growth rate, $k_{i}$. Thus, the eigenvalues of interest are located in the fourth quadrant, i.e. $k_{r}>0$ and $k_{i}<0$. The red circle depicts the non-spurious eigenvalue associated with the largest spatial growth rate and whose imaginary part corresponds to the red circle in Figure 1(c). This eigenvalue was distinguished among many spurious eigenvalues (out of the figure's visualization axis) by using the Gaster transformation of the temporal analysis, which has been used to obtain a target for the complex $k^{+}$spatial branches as explained in more detail in Iungo et al. (2013).

\subsection{Generic sensitivity analysis of the local stability problem}

The sensitivity of a given eigenvalue $k$ to generic perturbations of the linear operators in equation (2.4) is here derived by a standard expansion procedure. A small variation of the linear operators $\mathcal{C}_{0}^{\prime}=\mathcal{C}_{0}+\delta \mathcal{C}_{0}, \mathcal{C}_{1}^{\prime}=\mathcal{C}_{1}+\delta \mathcal{C}_{1}, \mathcal{C}_{2}^{\prime}=\mathcal{C}_{2}+\delta \mathcal{C}_{2}$ yields a small variation of the eigenvector $\hat{\mathbf{q}}^{\prime} \sim \hat{\mathbf{q}}+\delta \hat{\mathbf{q}}$ together with a small variation in the eigenvalue $k^{\prime} \sim k+\delta k$. At first order we have:

$$
\delta k=-\frac{<\mathbf{q}^{\dagger},\left[\delta \mathcal{C}_{0}+k \delta \mathcal{C}_{1}+k^{2} \delta \mathcal{C}_{2}\right] \hat{\mathbf{q}}>_{p}}{<\mathbf{q}^{\dagger},\left[\mathcal{C}_{1}+2 k \mathcal{C}_{2}\right] \hat{\mathbf{q}}>_{p}}
$$

where $\left\langle\cdot>_{p}\right.$ designates a suitable scalar product in the framework of parallel flows and $\mathbf{q}^{\dagger}=\left(\mathbf{u}^{\dagger}, p^{\dagger}\right)$ is the adjoint vector of the stability eigenproblem (2.4) associated with the 

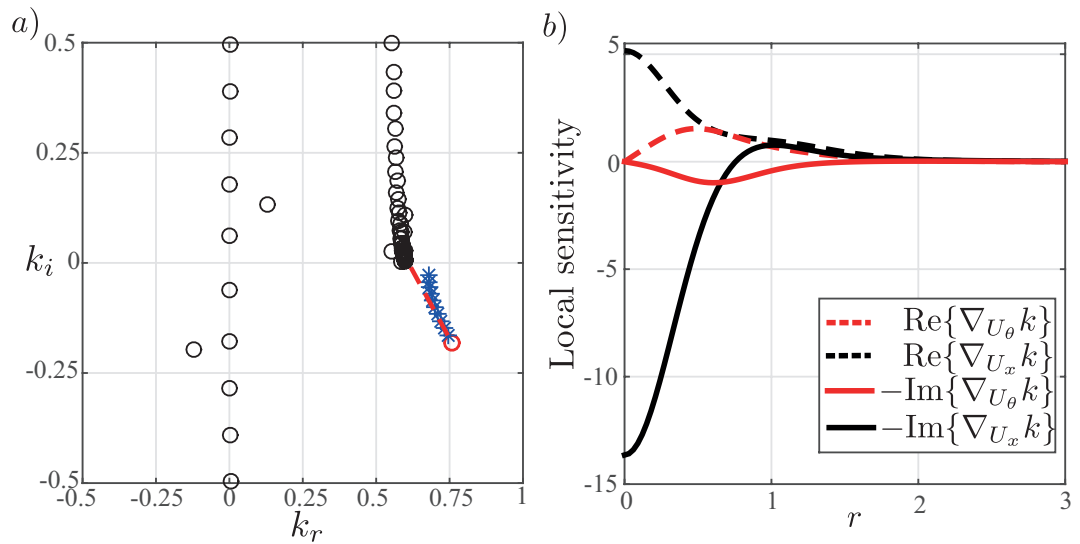

FiguRE 2. In (a) the circles are the spatial stability spectrum of the parallel Batchelor vortex with $\alpha=0.667, S=0.333, R e=100, m=1$ and $\omega=0.6$. The red circle indicates the most unstable physically meaningful eigenvalue. The blue asterisks mark the variation of the dominant eigenvalue (red circle) due to a modification of the base-flow velocity components, see text. The red line in (a) is the linear prediction of the eigenvalue variation based on the sensitivity map, whose components are reported in (b).

defined scalar product. From now on we will use the Hermitian inner product defined as

$$
<\mathbf{q}_{A}, \mathbf{q}_{B}>_{p}=\int_{0}^{\infty} \mathbf{q}_{A}^{H} \mathbf{q}_{B} r \mathrm{~d} r
$$

where the symbol ${ }^{H}$ indicates the trans-conjugate and $\mathbf{q}_{A}$ and $\mathbf{q}_{B}$ are two complex vector fields. In this case, the adjoint vector satisfies the following adjoint eigenvalue problem:

$$
k^{*^{2}} \mathcal{C}_{2}^{\dagger} \mathbf{q}^{\dagger}+k^{*} \mathcal{C}_{1}^{\dagger} \mathbf{q}^{\dagger}+\mathcal{C}_{0}^{\dagger} \mathbf{q}^{\dagger}=0
$$

where the superscript ${ }^{*}$ designates the complex conjugate and the adjoint operators $\mathcal{C}_{0}^{\dagger}, \mathcal{C}_{1}^{\dagger}$ and $\mathcal{C}_{2}^{\dagger}$ are reported in appendix $\mathrm{A}$. The adjoint vector $\mathbf{q}^{\dagger}$ is defined up to a multiplicative factor and from now the chosen normalization is such that the denominator of the expression (2.5) is equal to 1. Equation (2.5) is the generalization of the sensitivity of a standard eigenvalue problem described in Chomaz (2005), which would correspond to the case $\mathcal{C}_{2}=0$.

\subsection{Sensitivity of the local spatial stability properties to base flow modifications}

We are now interested in determining the sensitivity of $k$ to a generic base-flow modification $\delta \mathbf{U}=\left(\delta U_{r}=0, \delta U_{\theta}, \delta U_{x}\right)$, where the restriction $\delta U_{r}=0$ is dictated by the local analysis setting. A perturbation at the base-flow level in the linearized equation (2.2) yields a perturbation of the linear operators $\delta \mathcal{C}_{0}, \delta \mathcal{C}_{1}, \delta \mathcal{C}_{2}$ such that $\delta \mathcal{C}_{2}=0$ and

$$
\left[\delta \mathcal{C}_{0}+k \delta \mathcal{C}_{1}\right] \hat{\mathbf{q}}=\left(\begin{array}{c}
i m \hat{u}_{r} \delta U_{\theta} / r+i k \hat{u}_{r} \delta U_{x}-2 \hat{u}_{\theta} \delta U_{\theta} / r \\
\hat{u}_{r} \partial \delta U_{\theta} / \partial r+i m \hat{u}_{\theta} \delta U_{\theta} / r+i k \hat{u}_{r} \delta U_{x}+\hat{u}_{r} \delta U_{\theta} / r \\
\hat{u}_{r} \partial \delta U_{x} / \partial r+i m \hat{u}_{x} \delta U_{\theta} / r+i k \hat{u}_{x} \delta U_{x} \\
0
\end{array}\right) .
$$

Substituting equation (2.8) in equation (2.5) and integrating by parts we can transfer the differential operators from the vector $\delta \mathbf{U}$ to the adjoint vector $\mathbf{u}^{\dagger}$. If the base-flow modification does not occur at the domain boundaries, the boundary terms coming from the integration by parts are null and equation (2.5) reads:

$$
\delta k=\left\{\nabla_{\mathbf{U}} k, \delta \mathbf{U}\right\},
$$


where the complex vector

$$
\nabla_{\mathbf{U}} k=\left(\begin{array}{c}
0 \\
i m\left(u_{x}^{\dagger} \hat{u}_{x}^{H}+u_{r}^{\dagger} \hat{u}_{r}^{H}+u_{\theta}^{\dagger} \hat{u}_{\theta}^{H}\right)+\partial u_{\theta}^{\dagger} / \partial r \hat{u}_{r}^{H}+u_{\theta}^{\dagger} \partial \hat{u}_{r}^{H} / \partial r+2 u_{r}^{\dagger} \hat{u}_{\theta}^{H} / r \\
i k\left(u_{x}^{\dagger} \hat{u}_{x}^{H}+u_{r}^{\dagger} \hat{u}_{r}^{H}+u_{\theta}^{\dagger} \hat{u}_{\theta}^{H}\right)+\partial u_{x}^{\dagger} / \partial r \hat{u}_{r}^{H}+u_{x}^{\dagger} \partial \hat{u}_{r}^{H} / \partial r+u_{x}^{\dagger} \hat{u}_{r}^{H} / r
\end{array}\right)
$$

is the sensitivity of the considered spatial eigenvalue, $k$, to a base-flow modification. The azimuthal and streamwise components of $\nabla_{\mathbf{U}} k$ are the sensitivities of the complex eigenvalue $k$ to an azimuthal and streamwise base-flow modification. Their real (dashed line) and imaginary (full line) parts are reported in Figure 2 (b) for the most unstable spatial eigenvalue of the parallel Batchelor vortex in the case of $\alpha=0.667$ and $S=0.333$, i.e. $m=1$ and $\omega=0.6$. Since $\nabla_{\mathbf{U}} k$ is a complex vector and $\delta \mathbf{U}$ is a real quantity, the real part of $\nabla_{U_{\theta}} k$ and $\nabla_{U_{x}} k$, are the sensitivities of $k_{r}$ to a generic base-flow modification. On the other hand, the sensitivity of the spatial growth rate is given by the imaginary part of expression (2.10). The radial component of the sensitivity to base-flow is null consistently within the parallel flow assumption of the local analysis.

From the sensitivity maps in Figure 2 (b), it is seen that a base-flow modification with positive azimuthal and streamwise components superimposed to the reference baseflow (2.1) leads $k_{r}$ to increase. Moreover, a swirl increase and a decrease of the wake deficit yield a lower spatial growth-rate $-k_{i}$. Hence, equation (2.9) allows us to determine the direction of displacement of the spatial eigenvalues in the complex $k$-plane corresponding to a given base-flow modification. In order to verify the sensitivity maps we study the effect of a specific base-flow modification, i.e. $\delta U_{x}=c e^{-r^{2}}$, on the most unstable spatial eigenvalue in Figure 2 (a). The considered base-flow modification mimics a variation or uncertainty on the wake parameter $\alpha$ in equation (2.1) and its effect on the spatial stability is determined by solving the spatial stability analysis (2.4) with the modified base flow component $U_{x}+c e^{-r^{2}}$. Results are shown in Figure 2(a) for $c$ ranging from $3 \%$ to $30 \%$ of $\alpha$. The true path followed by the selected eigenvalue in the complex plane as the parameter $c$ is varied is indicated with blue asterisks. The same path is approximated by the red straight line using the sensitivity analysis carried out on the unperturbed flow. It is seen that the perturbed eigenvalue positions, which migrates towards the stability region as $c$ is increased, are well aligned with the linear prediction provided by sensitivity analysis, especially when $c$ is small, thus validating the method and its implementation. For larger base-flow modifications, nonlinearities neglected in the linearized relation (2.5), become more important and lead to a progressively increasing discrepancy between the predicted and the true eigenvalue trajectory.

Equation (2.10) is an extension to the spatial analysis of a concept that was originally developed by Bottaro et al. (2003) for temporal analysis. It should be also noted that the two non-null components of $\nabla_{U} k$ are equal to the azimuthal and axial components of the vector

$$
-\mathbf{u}^{\dagger} \cdot(\nabla \hat{\mathbf{u}})^{H}+\hat{\mathbf{u}}^{*} \cdot \nabla \mathbf{u}^{\dagger}
$$

which is formally similar to the sensitivity to base flow modifications of a global mode found by Marquet et al. (2008). The use of equation (2.11) in the framework of a local stability analysis requires $\hat{\mathbf{u}}$ to be the direct spatial mode, while $\mathbf{u}^{\dagger}$ is the spatial adjoint vector which satisfies the spatial adjoint problem (2.7). Moreover, the streamwise and azimuthal derivatives have to be replaced by the Fourier derivatives, according to the modal expansion (2.3). Finally, the radial component of (2.11), which would give the sensitivity to a radial velocity modification in a global framework, does not bear any meaning in the local spatial analysis. 


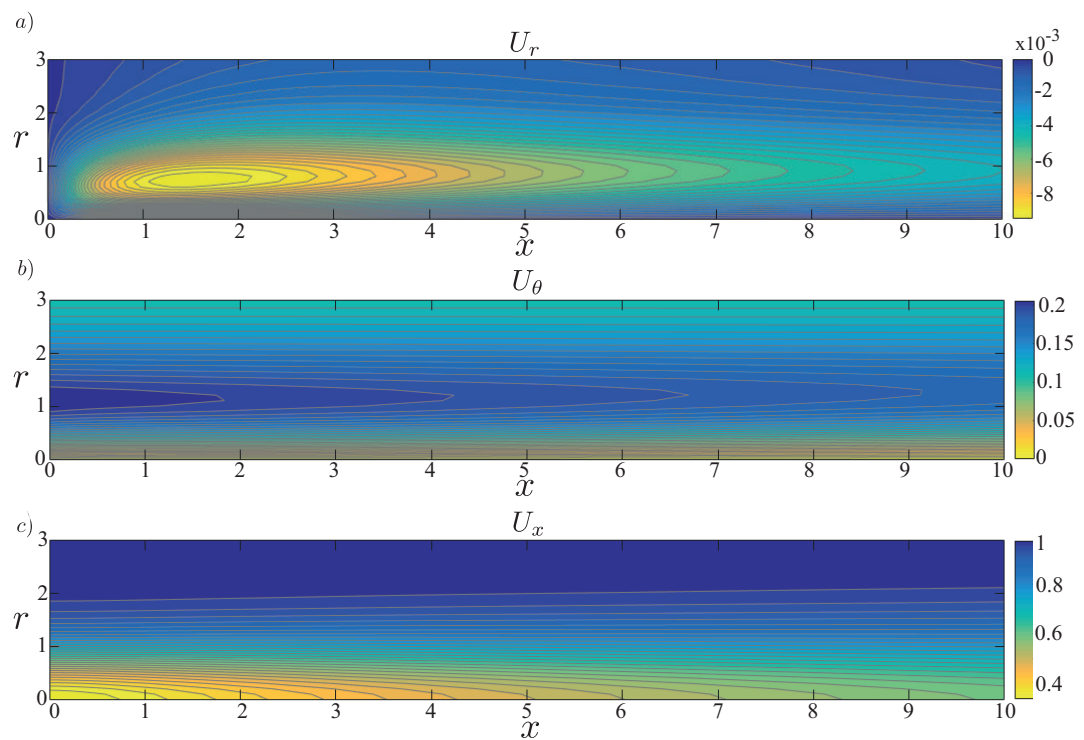

Figure 3. (a) Radial, (b) azimuthal and (c) streamwise velocity components of the non-parallel Batchelor vortex at $R e=100$ with inlet condition given by the velocity profile in equation (2.1).

\section{Uncontrolled trailing vortex prototype}

Rather than parallel flows, we now consider weakly non-parallel amplifier flows which slowly evolve in the streamwise direction, resulting in variations of the local stability properties. The non-parallel Batchelor vortex represents a suitable prototype flow, where its parallel approximation (2.1) considered in the previous section is here used as inlet condition together with free-stress condition at the outlet and radial far-field boundaries. Its velocity components and pressure $\mathbf{Q}=(\mathbf{U}, P)$ depend both an the radius $r$ and the streamwise distance $x$ from the inlet and are found by solving the discretized NavierStokes equations (3.1) in cylindrical coordinates:

$$
\mathbf{N}\left(\mathbf{Q}, \mathbf{U}_{0}\right)=\left(\begin{array}{c}
\mathbf{U} \cdot \nabla \mathbf{U}+\nabla P-\frac{1}{R e} \Delta \mathbf{U} \\
\nabla \cdot \mathbf{U}
\end{array}\right)=0,
$$

where the dependence from the inlet condition $\mathbf{U}_{0}$ is emphasized in the notation. For the numerical solution of equations (3.1), we have used a Newton-Raphson method based on a staggered pseudospectral Chebyshev-Chebyshev collocation method (Viola et al. 2016). The computational domain is $0<x<20$ and $0<r<10$ and it has been discretized by using 80 and 40 nodes in the streamwise and radial directions. The resulting velocity field at $R e=100$ is reported in Figure 3 (a,b,c). Note as in (a) the radial velocity is significantly smaller than the other two velocity components, thus validating the assumption of weakly non-parallel flow. Proceeding downstream the vortex core gets slightly diffused, as shown in (b), and the axial velocity deficit in (c) gradually recovers. The flow is seen to be locally convectively unstable and the resulting spatial growth rates, $-k_{i}$, are reported in Figure 4 as a function of the frequency, $\omega$ and the streamwise position, $x$. Consistently with the spatial stability properties of the inlet section (see Fig. 1(c)) four helical modes are amplified in the trailing vortex, namely $m \in\{-1,1,2,3\}$. The largest amplification is observed in the inlet region where the wake deficit is more pronounced. In particular, the single helical mode, $m=1$, has the largest spatial growth rate and is intense in the low frequency band, whereas the double helical mode, $m=2$, dominates for $0.8<\omega<1.3$. In addition, $m=3$ is the most amplified mode at high frequency and $m=-1$ is weakly 
8
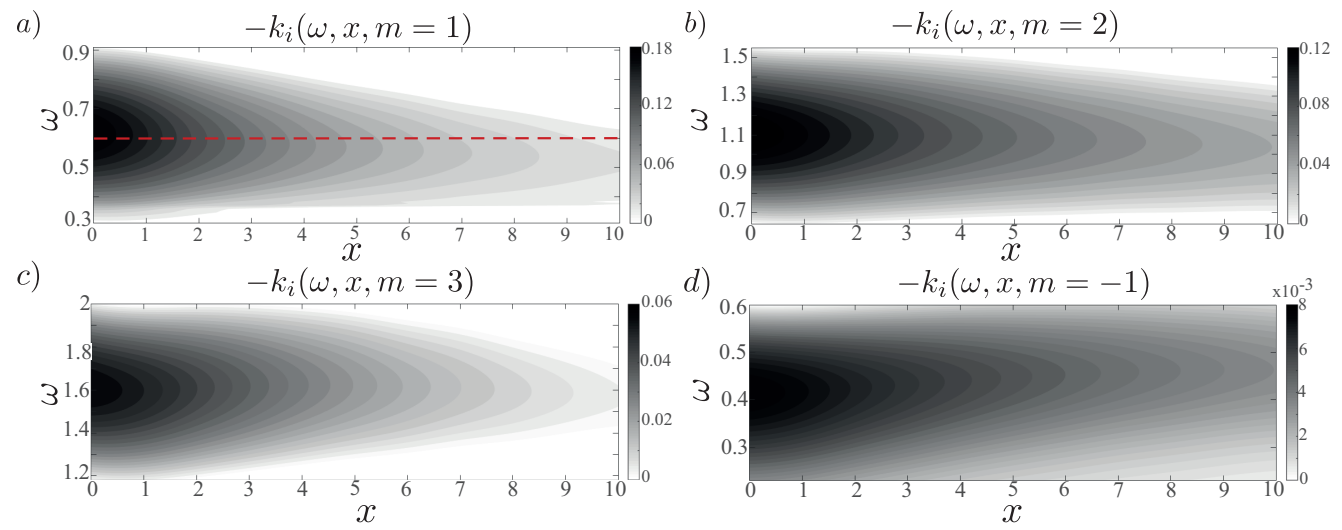

Figure 4. Contours of the spatial growth rate $-k_{i}$ in the $(\omega, x)$ plane for the convectively unstable helical modes, namely a) $m=1$, b) $m=2$, c) $m=3$ and d) $m=-1$. Each colorbar ranges from 0 (neutral amplification) to $\max \left(-k_{i}(\omega, x, m)\right.$ ). The horizontal dashed line in a) corresponds to the spatial branch examined in section 4.

amplified at low frequency.

Asymptotic theory for a weakly non-parallel base flow (Huerre \& Rossi 1998) prescribes that the global disturbance with frequency $\omega$ and azimuthal wavenumber mode $m$ takes the following modulated wave form at leading order:

$$
\mathbf{q}(r, \theta, x ; t) \sim \hat{\mathbf{q}}(r, x) \exp \left[i\left(\int_{0}^{x} k\left(x^{\prime}, \omega\right) d x^{\prime}+m \theta-\omega t\right)\right],
$$

where $k(x, \omega)$ is the local spatial eigenvalue at the flow location $x$ and $\hat{\mathbf{q}}(r, x)=(\hat{\mathbf{u}}, \hat{p})$ is the corresponding spatial eigenvector. In particular, the perturbation (3.2) is amplified or damped in space according to the local spatial growth rate $-k_{i}(x)$ and the integral amplification factor

$$
G(\omega, m)=\exp \left(\int_{0}^{x_{f}}-k_{i}\left(x^{\prime}, \omega, m\right) \mathrm{d} x^{\prime}\right),
$$

is a measure of the ratio between the final, $x_{f}$, and the initial, $x=0$, disturbance amplitudes, where $x_{f}$ has been set equal to $x_{f}=10 . G(\omega, m)$, which is obtained by integrating in $x$ the spatial growth rates in Figure (4), is reported in Figure 9(b) by a black line: the azimuthal geometry of the most amplified mode depends on the disturbance frequency, with $m=1$ well resonating around $\omega=0.6$ and $m=2$ around $\omega=1.1$.

Furthermore, we define the total amplification factor as

$$
\Gamma=\sum_{m \in \mathcal{M}} \int_{\omega_{\min }}^{\omega_{\max }} G(\omega, m) \mathrm{d} \omega,
$$

where $\left[\omega_{\min }, \omega_{\max }\right]$ is the frequency band of noise amplification (here equal to $[0,2.1]$, see Fig. $9(\mathrm{~b}))$ and $\mathcal{M}$ is the set of convectively unstable helical modes, namely $\mathcal{M}=$ $\{-1,1,2,3\}$. $\Gamma$ represents the overall amplification of all modes at all frequencies after they have undergone their entire amplification process in space between 0 and $x_{f}$.

In what follows the sensitivity of the integral (3.3) and total (3.4) amplification factors to an inflow modification is derived by using the results presented in section 2.3 , thus providing information for their control. 
a)

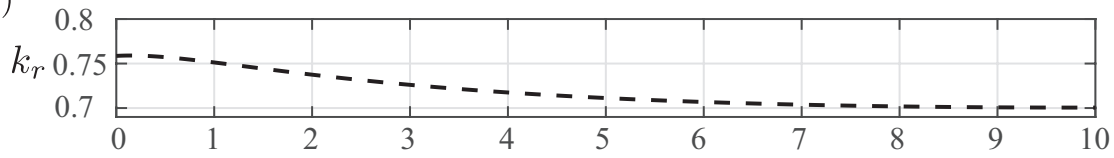

b)

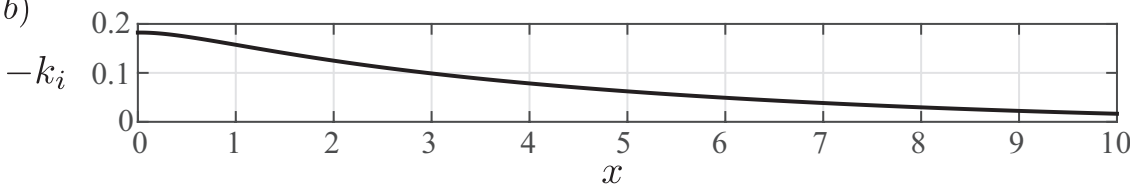

FIgURE 5. Streamwise evolution of the (a) axialwavenumber, $k_{r}$, and of the (b) spatial growth rate, $-k_{i}$, for the single-helix spatial branch at $\omega=0.6$.

\section{Sensitivity of the integral amplification factor in weakly nonparallel flows}

\subsection{Lagrangian formulation}

In this section, specifically for control purposes, we are interested in determining the sensitivity of the integral amplification factor (3.3) of a specific spatial mode. As a reference case, we consider the streamwise evolution of the single helical mode $m=1$ at frequency $\omega=0.6$ in the evolving Batchelor vortex (see section 3) which is the most amplified mode in the domain and which corresponds to the horizontal dashed line in Figure 4(a). By carrying out the local spatial analysis at different positions in the $x$ direction, it is seen that both the axial wavenumber $k_{r}$ and the spatial growth rate, $-k_{i}$, decrease while moving downstream due to the reduction of the wake deficit, see Figure 5.

This control problem can be formulated as an optimization problem where the objective function is the integral amplification itself. Rather than a continuous approach where the continuous optimization problem is defined and then discretized, we directly apply the Lagrangian variational technique to the discretized governing equations:

$$
\mathscr{L}_{G}\left(\mathbf{Q}, \mathbf{U}_{0}\right)=G(\mathbf{Q})+\boldsymbol{\lambda}^{T} \mathbf{N}\left(\mathbf{Q}, \mathbf{U}_{0}\right),
$$

where the superscript ${ }^{T}$ stands for the transpose. The constraint is given by the discretized steady Navier-Stokes equations, $\mathbf{N}\left(\mathbf{Q}, \mathbf{U}_{0}\right)$, and $\boldsymbol{\lambda}$ is the vector of Lagrange multipliers or discrete adjoint vector. Thus, the integral amplification factor (3.3) is written in discretized form as follows:

$$
G(\omega, m)=\exp \left(-\sum_{n=1}^{N_{x}} w_{n} k_{i}\left(x_{n}, \omega, m\right)\right) .
$$

In equation (4.2) the coefficients $w_{n}$ are the integration weights of the standard trapezoidal formula and $N_{x}$ is the number of locations used to discretize the streamwise direction.

The gradient of the integral amplification factor $G$ with respect to a base-flow inlet modification is derived by considering variations of the Lagrangian $\mathscr{L}_{G}$. Imposing the stationarity of $\mathscr{L}_{G}$ with respect to $\boldsymbol{\lambda}$ reduces to the discrete state equation (3.1), which is satisfied by the uncontrolled base-flow $\mathbf{Q}$. Then, the stationarity of the Lagrangian with respect to the state variable, $\mathbf{Q}$, yields an equation which has to be satisfied by the adjoint variable $\boldsymbol{\lambda}$ :

$$
-\boldsymbol{\lambda}^{T} \nabla_{\mathbf{Q}} \mathbf{N}=\nabla_{\mathbf{Q}} G
$$

where $\nabla_{\mathbf{Q}} \mathbf{N}$ is the Jacobian matrix of the discrete Navier-Stokes operator. Recalling that the local growth rates $-k_{i}\left(x_{n}\right)$ depend only on the streamwise and azimuthal velocity at 
the location $x_{n}$, the rhs of equation (4.3) can be rewritten as follows

$$
\nabla_{\mathbf{Q}} G=-G \sum_{n=1}^{N_{x}} w_{n}\left[\nabla_{U_{x}} k_{i}\left(x_{n}\right) \nabla_{\mathbf{Q}} U_{x}\left(x_{n}\right)+\nabla_{U_{\theta}} k_{i}\left(x_{n}\right) \nabla_{\mathbf{Q}} U_{\theta}\left(x_{n}\right)\right],
$$

where the terms $\nabla_{U_{x}} k_{i}\left(x_{n}\right)$ and $\nabla_{U_{\theta}} k_{i}\left(x_{n}\right)$ are the local spatial sensitivities of $k_{i}$ to a local base-flow modification and the related expressions have been already derived in section 2.3. Specifically, $\nabla_{U_{x}} k_{i}\left(x_{n}\right)$ and $\nabla_{U_{\theta}} k_{i}\left(x_{n}\right)$ have to be evaluated by injecting the local direct $\hat{\mathbf{u}}\left(x_{n}\right)$ and adjoint $\mathbf{u}^{\dagger}\left(x_{n}\right)$ modes in equation (2.10). The terms $\nabla_{\mathbf{Q}} U_{x}\left(x_{n}\right)$ and $\nabla_{\mathbf{Q}} U_{\theta}\left(x_{n}\right)$ are the interpolation matrices which extract from a vector $\mathbf{Q}$ the streamwise and azimuthal velocity components at the streamwise position $x_{n}$. Hence, given the local spatial sensitivity analyses, the adjoint vector $\boldsymbol{\lambda}$ can be determined by solving the linear system (4.3).

Finally, the derivative of the Lagrangian $\mathscr{L}_{G}$ with respect to the control variable gives the variation of the integral amplification factor with respect to a small-amplitude generic inlet modification $\delta \mathbf{U}_{0}$ :

$$
\delta G=\frac{d G(\mathbf{Q})}{d \mathbf{U}_{0}} \delta \mathbf{U}_{0}=\boldsymbol{\lambda}^{T} \nabla_{\mathbf{U}_{0}} \mathbf{N} \delta \mathbf{U}_{0}
$$

where $\nabla_{\mathbf{U}_{0}} \mathbf{N}$ is a restriction matrix, which extracts the velocity components at the inlet $x=0$ from a velocity-pressure vector $\mathbf{Q}$. Equation (4.5) can be formally rewritten as

$$
\delta G=<\nabla_{\mathbf{U}_{0}} G, \delta \mathbf{U}_{0}>
$$

where $<\mathbf{u}_{A}, \mathbf{u}_{B}>=\mathbf{u}_{A}^{T} M \mathbf{u}_{B}$ is the inner product in the discrete setting, and $M$ is the integration matrix containing the integration weights on its diagonal. The real vector $\nabla_{\mathbf{U}_{0}} G$ is the integral amplification factor sensitivity with respect to an inlet modification, and is defined as:

$$
\nabla_{\mathbf{U}_{0}} G=M^{-1}\left(\nabla_{\mathbf{U}_{0}} \mathbf{N}\right)^{T} \boldsymbol{\lambda}
$$

For the reference case of $\omega=0.6$ and $m=1$, the three components of $\nabla_{\mathbf{U}_{0}} G$, which correspond to the sensitivity maps to a radial, azimuthal and axial inlet velocity modification, are reported in Figure 6(a). The sensitivity with respect $U_{\theta_{0}}$ reveals that in order to increase the amplification, the azimuthal velocity, and therefore the swirl parameter, has to be decreased. Similarly, $\nabla_{U_{x_{0}}} G$ shows that an increase of the wake deficit and a higher streamwise velocity at the vortex periphery lead to a more intense response to noise. Due to the nonparallelism of the base-flow $\mathbf{U}$, the spatial amplification is also sensitive to a variation of the radial velocity. This velocity component acts on the local spatial stability properties of the flow solely through the coupling with the streamwise and azimuthal velocity components in the base-flow governing equations (3.1). In particular it is seen that a positive inlet radial velocity, which increases the vortex core diameter and consequently favors the axial deceleration, has a destabilizing effect.

Similar results on the sensitivity with respect to radial and axial velocity components are observed for $m=2$ and $m=3$ at the frequency corresponding to the largest amplification factor, $\omega=1.1$ and $\omega=1.6$ respectively, see Figure 7 . In contrast to $m=1$ (Fig. 6(a)), however, the sensitivity with respect to the azimuthal velocity is positive in the core region for both $m=2$ and $m=3$. This signifies that an increase of the swirl has a destabilizing effect on the double and triple helical modes. This result can be rationalized by recalling that, starting from a pure axial flow, helical modes are first progressively destabilized by an increasing amount of swirl till $S$ exceeds $S^{*}(m)$, beyond which a further increase of swirl starts to have a damping effect, until complete stabilization of the flow for $S \approx 1.5 \alpha$ (Mayer \& Powell 1992; Delbende et al. 1998). As illustrated 

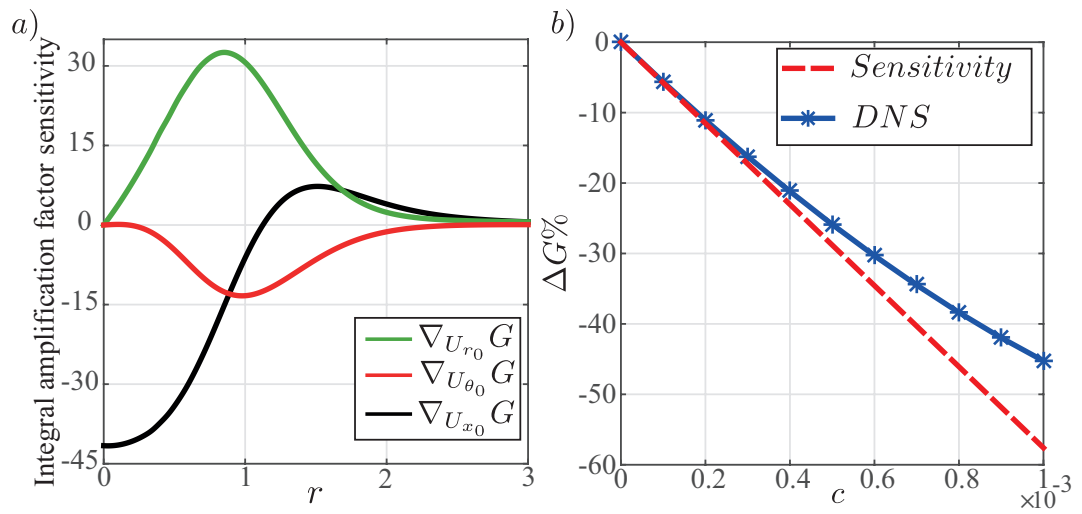

FiguRE 6. In (a) the sensitivity maps of $G(\omega=0.6, m=1)$ to a radial (green), azimuthal (red) and axial (black) inlet velocity modifications. In (b) the true amplification factor modification is reported with blue asterisks as a function of the magnitude of the inlet control $c$. The red dashed line shows the linear amplification modification according to the inlet sensitivity map.
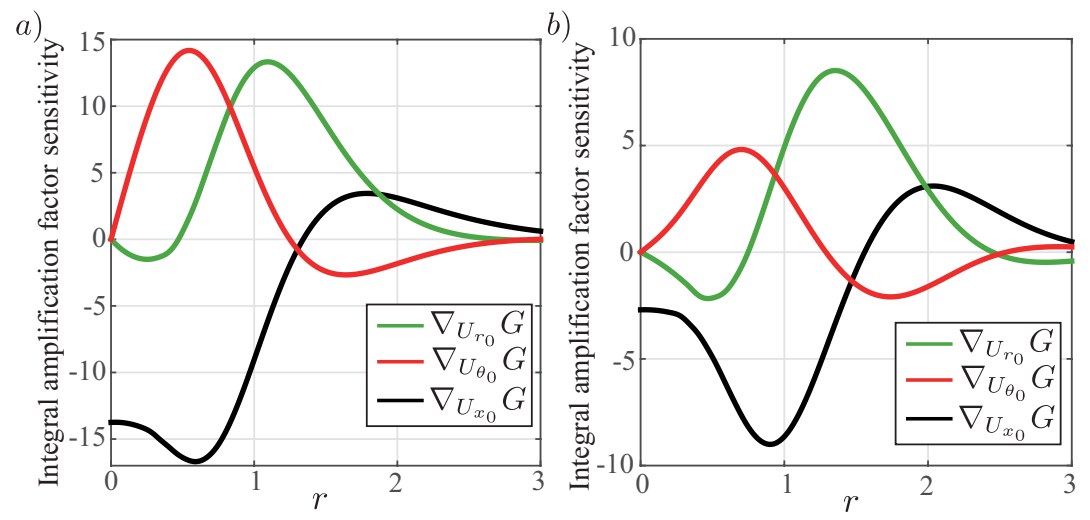

FiguRE 7. Sensitivity maps of a) $G(\omega=1.1, m=2)$ and b) $G(\omega=1.6, m=3)$ to a radial (green), azimuthal (red) and axial (black) inlet velocity modifications.

in Figure 1(b) for the flow pertaining at the inlet section, the swirl value corresponding to the maximum growth rate, $S^{*}(m)$, depends on the helical mode and it is seen to increase with $m$ for the chosen flow parameters, thus yielding a different sensitivity to a modification in the inlet azimuthal velocity.

\subsection{Optimal inlet control}

The sensitivity $\nabla_{\mathbf{U}_{0}} G$ not only predicts the linear modification of the integral amplification factor due to an inlet modification through (4.6), but it also corresponds to the most destabilizing infinitesimal inlet modification (Camarri 2015). For this reason, $\nabla_{\mathbf{U}_{0}} G$ can be used to design an inlet control which stabilizes the global spatial branch. Figure 6(b) shows the amplification variation of the single-helix spatial branch at frequency $\omega=0.6$ due to an inlet modification of the type $\delta \mathbf{U}_{0}=-c \nabla_{\mathbf{U}_{0}} G$, with $c$ the magnitude of the control. The blue line in that figure represents the real amplification variation with respect to the uncontrolled condition. More precisely, at a given $c$, the real amplification variation (blue asterisk) is computed by (i) solving the non-linear base flow equations (3.1) with the inlet condition $\mathbf{U}_{0}-c \nabla_{\mathbf{U}_{0}} G$, and (ii) carrying out the spatial stability analysis on the obtained base-flow. Then, (iii) the associated integral amplification factor $G(c)$ is 
12

a)

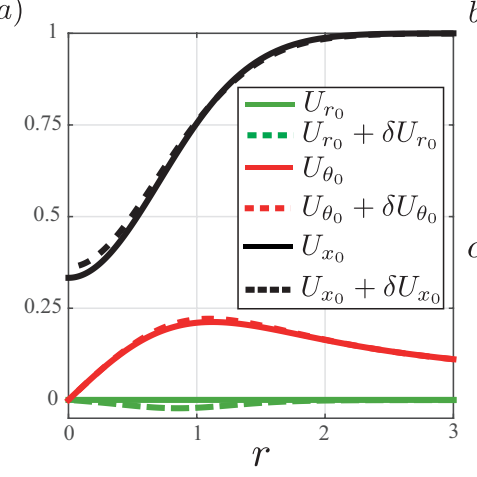

b)

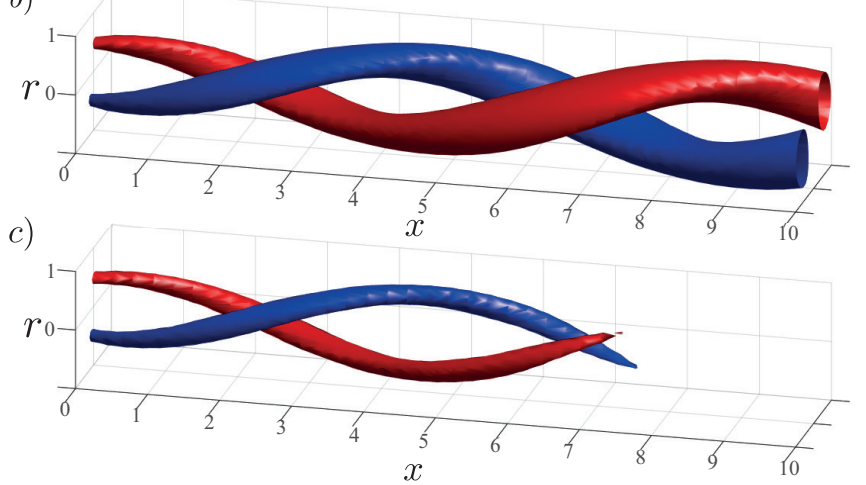

FiguRE 8. (a) Full lines indicate the velocity components of the uncontrolled inlet condition, which correspond to the Batchelor vortex profile (2.1) with $\alpha=0.667$ and $S=0.333$. The velocity components perturbed by the application of the control $\delta \mathbf{U}_{0}=7 \cdot 10^{-4} \nabla_{\mathbf{U}_{0}} G(\omega=0.6, m=1)$ are shown with dashed line. (b) Isosurfaces of $\pm 95 \%$ of the maximum axial vorticity of the global spatial mode (3.2) in the (b) uncontrolled and (c) controlled case.

computed according to the definition (4.2) and its variation $\Delta G=1-G(c) / G(c=0)$ is obtained, where $G(c=0)$ is the amplification factor of the uncontrolled condition. It is seen that the linear amplification variation predicted by equation (4.6), which is depicted by a red line in Fig. 6(b), correctly captures the amplification variation for small values of $c$. In contrast, due to the nonlinear dependence of the amplification factor on the magnitude of the perturbation the error increases as $c$ is progressively increased. Still, the linearization leading to the sensitivity analysis allows the prediction of even large variations of amplification with reasonable accuracy.

As an application, Figure 8(a) shows the uncontrolled and the controlled velocity profiles at the inlet for $c=7 \cdot 10^{-4}$ (see Fig. 6(a)). Although the inlet condition differs very slightly form the uncontrolled case, the effect of this variation on the considered instability is substantial as shown in Fig. 8(b) and (c) where the axial vorticity of the spatial branch given by equation (3.2) with $\omega=0.6$ and $m=1$ is reported. In the first case the helical perturbation is convectively unstable in all the flow domain and is continuously amplified while propagating. When the control is applied, the spatial branch is damped for $x>7$ and the integral amplification factor experiences a significative reduction of $35 \%$ with respect to the uncontrolled flow.

\section{Sensitivity of the total growth factor in weakly nonparallel flows}

\subsection{Lagrangian formulation}

The control strategy illustrated in the previous section is based on the inlet sensitivity of the integral amplification factor of a single helical mode at a given frequency. Hence, a control is designed to reduce the amplification of the single spatial branch considered, but it could dangerously increase the spatial growth of other helical modes and of other frequencies. Therefore, we now turn to assess the sensitivity of the total amplification factor $\Gamma$ (see equation (3.4)) to a modification of the inflow conditions. Indeed, the control of $\Gamma$ requires to account for the local growth rates of all helical modes in their entire frequency band of amplification.

Similarly to the analysis in section 4.1 , the problem can be tackled by considering the 
following Lagrangian:

$$
\mathscr{L}_{\Gamma}\left(\mathbf{Q}, \mathbf{U}_{0}\right)=\Gamma(\mathbf{Q})+\boldsymbol{\xi}^{T} \mathbf{N}\left(\mathbf{Q}, \mathbf{U}_{0}\right) .
$$

The steady Navier-Stokes equations $\mathbf{N}\left(\mathbf{Q}, \mathbf{U}_{0}\right)$ is the constraint, the Lagrange multiplier $\boldsymbol{\xi}$ is the adjoint vector and the quantity to minimize is now the total amplification factor $\Gamma(\mathbf{Q})$, which in discrete form reads:

$$
\Gamma=\sum_{m \in \mathcal{M}} \sum_{l=1}^{N_{\omega}} \alpha_{l} G\left(\omega_{l}, m\right)=\sum_{m \in \mathcal{M}} \sum_{l=1}^{N_{\omega}} \alpha_{l} \exp \left(-\sum_{n=1}^{N_{x}} w_{n} k_{i}\left(x_{n}, \omega_{l}, m\right)\right) .
$$

As in equation (4.2), the coefficients $w_{n}$ are the integration weights of the standard trapezoidal formula and $N_{x}$ is the number of locations used to discretize the control window in the streamwise direction. In addition, the coefficient $\alpha_{l}$ are the integration weights corresponding to the integral in the frequency domain, which is discretized using $N_{\omega}$ points and $\mathcal{M}=\{-1,1,2,3\}$ is the set of convectively unstable helical modes.

When imposing the stationarity of $\mathscr{L}_{\Gamma}$ with respect to $\boldsymbol{\xi}$, the problem reduces to the discrete state equation (3.1), which is satisfied by the uncontrolled base-flow $\mathbf{Q}$ and that we have determined previously. Then, the stationarity of the Lagrangian with respect to the state variable, $\mathbf{Q}$, yields a governing equation for the adjoint variable $\boldsymbol{\xi}$

$$
-\boldsymbol{\xi}^{T} \nabla_{\mathbf{Q}} \mathbf{N}=\nabla_{\mathbf{Q}} \Gamma
$$

which is now forced at the right-hand-side by the variation of the total amplification factor, $\Gamma$, with respect to a variation of the baseflow, $\mathbf{Q}$. This term can be rewritten as a function of the sensitivities to a base-flow variation of the helical modes' amplification factors at different frequencies,

$$
\nabla_{\mathbf{Q}} \Gamma=\sum_{m \in \mathcal{M}} \sum_{l=1}^{N_{\omega}} \alpha_{l} \nabla_{\mathbf{Q}} G(\omega, m),
$$

which reduces, by using equation (4.4), to a weighted sum of the local spatial sensitivities to a generic local base-flow modification (see section 2.3):

$$
\nabla_{\mathbf{Q}} \Gamma=\sum_{m \in \mathcal{M}} \sum_{l=1}^{N_{\omega}}-\alpha_{l} G(\omega, m) \sum_{n=1}^{N_{x}} w_{n}\left[\nabla_{U_{x}} k_{i}\left(x_{n}\right) \nabla_{\mathbf{Q}} U_{x}\left(x_{n}\right)+\nabla_{U_{\theta}} k_{i}\left(x_{n}\right) \nabla_{\mathbf{Q}} U_{\theta}\left(x_{n}\right)\right] .
$$

Note that the local sensitivities $\nabla_{U_{\theta}} k_{i}\left(x_{n}\right)$ and $\nabla_{U_{x}} k_{i}\left(x_{n}\right)$ in equation (5.5) are multiplied by the integral amplification factor of the given spatial branch $G(\omega, m)$. Hence, as reasonable, most amplified helical modes correspond to a higher contribution to the forcing term $\nabla_{\mathbf{Q}} \Gamma$. Then, by solving the linear system (5.3) for the adjoint field $\boldsymbol{\xi}$, the sensitivity of the total amplification factor with respect a small-amplitude generic inlet modification $\delta \mathbf{U}_{0}$ reads:

$$
\nabla_{\mathbf{U}_{0}} \Gamma=M^{-1}\left(\nabla_{\mathbf{U}_{0}} \mathbf{N}\right)^{T} \boldsymbol{\xi} .
$$

The three components of the sensitivity $\nabla_{\mathbf{U}_{0}} \Gamma$ are shown in Figure 9 (a) and they correspond to the sensitivity maps to a radial (green line), azimuthal (red line) and axial (black line) inlet velocity modification.

Similarly to the sensitivity maps in Figure 6(a) and 7, where only one helical mode at a given frequency was considered, an increase of the wake deficit and a higher streamwise velocity at the vortex periphery leads to a more intense amplification of disturbances. Moreover, a positive inlet radial velocity is positively correlated with $\delta \Gamma$. In contrast, the sensitivity with respect to a modification of the azimuthal velocity has not a monotonic 
a)

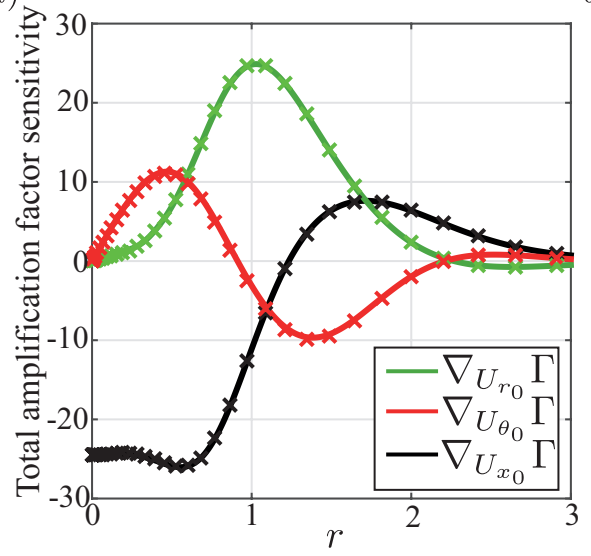

b)

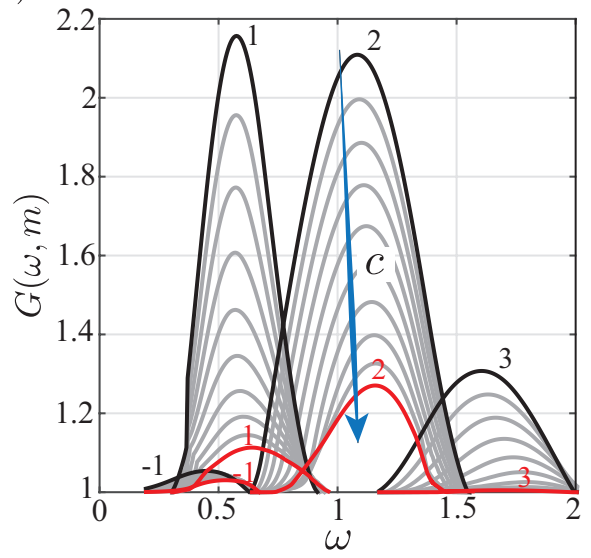

Figure 9. Full lines in (a) show the sensitivity maps of the total amplification factor $\Gamma$ (see equation (5.6)) to a radial (green), azimuthal (red) and streamwise (black) inlet velocity modifications. The + symbols correspond to the sensitivity maps computed with a finer mesh (see appendix B), showing convergence with respect to grid resolution. The variation of the integral amplification factor $G(\omega, m)$ obtained by using the sensitivity map as inlet control $\delta \mathbf{U}_{0}=c \nabla_{\mathbf{U}_{0}} \Gamma$ is shown in (b). Gray lines correspond to several forcing amplitudes $c$ ranging from $c=0$ (uncontrolled condition, black line) to $c=2 \cdot 10^{-3}$ (red line) in equal intervals.

behaviour since it is the result of a compromise between the sensitivities of different helical modes. As previously discussed in section 4.1, double and triple helical modes are destabilized by an increase of the azimuthal velocity close to the centerline, see Figure 7 . On the other hand, the single helical mode requires a decrease in swirl to enhance the amplitude of the response, as depicted in Figure 6(a).

\subsection{Optimal inlet control}

The sensitivity map (5.6) is now used as an inlet control to reduce the integral amplification factor $G(\omega, m)$ of all helical modes over the base-flow. Figure $9(\mathrm{~b})$ shows the variation of $G(\omega, m)$ due to an inlet modification of the type $\delta \mathbf{U}_{0}=-c \nabla_{\mathbf{U}_{0}} \Gamma$, with $c$ the magnitude of the control. Gray lines depict the dependence of $G(\omega, m)$ on the control amplitude ranging from the uncontrolled condition $c=0$ (black line) to $c=2 \cdot 10^{-3}$ in equal intervals. At a given $c$, the integral amplification factor is obtained by (i) solving the non-linear base flow equations (3.1) with the inlet condition $\mathbf{U}_{0}-c \nabla_{\mathbf{U}_{0}} \Gamma$, and (ii) carrying out the spatial stability analysis on the obtained base-flow.

The figure demonstrates how with a single linearization of the governing equation is possible to design a control strategy able to reduce the spatial amplification of all convectively unstable disturbances. In particular, with an amplitude of the control of $c=2 \cdot 10^{-3}$ (red line) the integral amplification factor is reduced by $50 \%$ for $m=1$ and by $40 \%$ for $m=2$ and higher azimuthal wavenumber modes are seen not to be destabilized by the control. Moreover, the high frequency mode $m=3$ is completely stabilized and $m=-1$, which was already weakly amplified, is slightly stabilized.

Note that the control is more effective on $m=1$ rather than $m=2$, which progressively becomes the most amplified mode while the control amplitude $c$ is increased. This behaviour can be explained by recalling that the control is designed on the uncontrolled base-state where $m=1$ has the largest $G(\omega, m)$ and therefore has the highest contribution to the forcing term (5.5) and on the sensitivity map. However, the sensitivity maps defined here can be used in an iterative shaping of the control as proposed by Camarri \& 
Iollo (2010). This technique, which is based on successive linearizations of the governing equations, allows to properly modify the control according to the new stability property of the controlled base-flow.

\section{Conclusions}

In this work a general formulation is proposed which allows the application of systematic adjoint-based techniques for the control of the integral amplification factor in weakly non-parallel amplifier flows. The method is based on the sensitivity analysis of the local stability properties of the flow, which was here derived by adjoint methods.

Firstly the sensitivity of the spatial stability spectrum of a locally parallel flow to a generic modification of the stability operators and to a base flow modification is found. In the case of a parallel Batchelor vortex, the local sensitivity map is seen to correctly predict the displacement of the spatial eigenvalues in the complex $k$-plane due to a specific base-flow modification.

Then, for the purpose of control in slowly evolving amplifier flows, these local results are used to determine the sensitivity of the spatial growth of disturbances to a modification of the inflow conditions. Although the method can be easily extended to cover very general cases, we presented here its application to the control of the spatial instability in an evolving Batchelor vortex that is traditionally used to model trailing vortices in aeronautics. The base-flow is obtained by axisymmetric direct numerical simulation starting from the velocity profile of a parallel Batchelor vortex as inflow boundary condition, and is seen to be locally convectively unstable to four helical modes, $m \in\{-1,1,2,3\}$. The largest amplification is observed in the inlet region where the wake deficit is more pronounced and the single and double helical modes are the most amplified modes.

The sensitivity of the integral amplification factor, $G(\omega, m)$, of a single spatial branch is formulated as an optimization problem where $G(\omega, m)$ is the objective function and the Navier-Stokes equations are included in the Lagrangian formulation as a constraint. Rather than a continuous approach where the continuous optimization problem is defined and then discretized, we directly apply the Lagrangian variational technique to the discretized governing equations. In this framework, we show that the local spatial sensitivities are the building blocks of the sensitivity of the integral amplification factor to generic modifications of the inlet velocity profile. The resulting sensitivity map indicates the optimal modification of the inlet velocity profile so as to stabilize the considered helical spatial mode of a non-parallel Batchelor vortex, which is locally convectively unstable. It is shown that the control, formulated using only one linearization of the flow dynamics carried out on the uncontrolled configuration, successfully reduces the global spatial amplification of a given spatial branch.

This analysis is then extended to determine the sensitivity to an inflow modification of the total amplification factor which is a measure of the overall amplification of all the convectively unstable spatial branches. Hence, the corresponding sensitivity map accounts for all the amplified helical modes in the frequency band of amplification. It is seen that, in order to increase the total amplification factor, the streamwise velocity has to decrease close to center of the wake and to increase at the vortex periphery, thus enhancing the wake deficit. A positive variation of the azimuthal velocity has a destabilizing effect close to the vortex centerline whereas it stabilizes the flow in the outer region. This non-monotonous behaviour is seen to be the result of the different sensitivities to the base-flow swirl of different helical modes. Furthermore, due to the non-parallelism of the base-flow, also the radial velocity has an effect on the spatial amplification: a positive inlet radial velocity increases the vortex core diameter favoring the axial deceleration and 
destabilizing the flow. This map is subsequently used as an inlet control to effectively reduce the integral amplification factor of all helical modes over the base-flow. We observe that with a control of size $c=2 \%$ o the spatial amplification of all modes is significantly reduced, and the amplification factor of $m=1$ and $m=2$ is almost halved.

The information provided by the control map is valid only for small control amplitudes perturbing the uncontrolled configuration. However, the control design method proposed here can be easily extended to include larger control amplitudes, by employing the sensitivity map in a standard iterative gradient method, as done for instance by Bottaro et al. (2003) for temporal analysis on parallel flow. We conclude underlying that the systematic framework proposed here to control the integral amplification factor in amplifier flows has potential impact in many cases of interest such as the control of the hub vortex in wind turbines (Iungo et al. 2013), which has inspired this work. As a final note, in the spirit of weakly non-parallel flows, the full Navier-Stokes equations in the Lagrangian formulation (4.1) can be replaced by the parabolized equations in the streamwise direction, allowing a marching technique for their numerical solution as for the boundary layer equations, see Zuccher et al. (2006). In this case, both the base-flow and stability problems are carried out in a weakly non-parallel setting.

\section{Appendix A. Local stability analysis of swirling flows}

The linear evolution of helical disturbances of the type $(\hat{\mathbf{u}}, \hat{p}) e^{i(k x+m \theta-\omega t)}$ over a parallel swirling flow $U_{x}(r), U_{\theta}(r)$ is governed by

$$
\begin{aligned}
& -i \omega \hat{u}_{r}+\Lambda_{m, k} \hat{u}_{r}-2 \frac{U_{\theta} \hat{u}_{\theta}}{r}=-\frac{\partial \hat{p}}{\partial r}+\frac{1}{R e}\left[\frac{\partial}{\partial r}\left(\frac{1}{r} \frac{\partial r \hat{u}_{r}}{\partial r}\right)-\left(m^{2} / r^{2}+k^{2}\right) \hat{u}_{r}-2 i m \frac{\hat{u}_{\theta}}{r^{2}}\right] \\
& -i \omega \hat{u}_{\theta}+\Lambda_{m, k} \hat{u}_{\theta}+\frac{\hat{u}_{r}}{r} \frac{\partial r U_{\theta}}{\partial r}=-\frac{i m}{r} \hat{p}+\frac{1}{R e}\left[\frac{\partial}{\partial r}\left(\frac{1}{r} \frac{\partial r \hat{u}_{\theta}}{\partial r}\right)-\left(m^{2} / r^{2}+k^{2}\right) \hat{u}_{\theta}+2 i m \frac{\hat{u}_{r}}{r^{2}}\right] \\
& -i \omega \hat{u}_{x}+\Lambda_{m, k} \hat{u}_{x}+\hat{u}_{r} \frac{\partial U_{x}}{\partial r}=-i k \hat{p}+\frac{1}{R e}\left[\frac{1}{r} \frac{\partial}{\partial r}\left(r \frac{\partial \hat{u}_{x}}{\partial r}\right)-\left(m^{2} / r^{2}+k^{2}\right) \hat{u}_{x}\right] \\
& \frac{1}{r} \frac{\partial r \hat{u}_{r}}{\partial r}+\frac{i m}{r} \hat{u}_{\theta}+i k \hat{u}_{x}=0,
\end{aligned}
$$

where $\Lambda_{m, k}=U_{\theta} i m / r+U_{x} i k$ is the base-flow advection operator. Homogeneous Neumann boundary conditions are imposed at the lateral boundary $r_{\max }$ together with the regularity conditions on the axis proposed by Batchelor \& Gill (1962):

$$
\begin{aligned}
& \hat{u}_{r}=\hat{u}_{\theta}=\frac{\partial \hat{u}_{x}}{\partial r}=0 \quad \text { for } m=0, \\
& \frac{\partial \hat{u}_{r}}{\partial r}=\frac{\partial \hat{u}_{\theta}}{\partial r}=\hat{u}_{x}=0 \quad \text { for }|m|=1 \\
& \hat{u}_{r}=\hat{u}_{\theta}=\hat{u}_{x}=0 \quad \text { for }|m|>1 .
\end{aligned}
$$

Temporal stability analysis allows the investigation of the temporal evolution of the helical disturbances, see Schmid \& Henningson (2012). In this framework, the axial wavenumber $k$ is real and $\omega$ is complex. Its real part, $\omega_{r}$, is the frequency of the perturbation and its imaginary part, $\omega_{i}$, is the temporal growth rate. Given the azimuthal, $m$, and streamwise, $k$, wavenumbers, equations (A 1 ) reduce to a generalized eigenvalue problem,

$$
\mathcal{A} \hat{\mathbf{q}}=\omega \mathcal{B} \hat{\mathbf{q}},
$$


where $\omega$ is the eigenvalue and the linear operators are defined as follows:

$$
\begin{gathered}
\mathcal{A}=\left[\begin{array}{cccc}
\Lambda_{m, k}-\frac{1}{R e} \Delta_{m, r \theta x}^{*} & -2 U_{\theta} / r+\frac{1}{R e} \frac{2 i m}{r^{2}} & 0 & \frac{\partial}{\partial r} \\
\frac{\partial U_{\theta}}{\partial r}+U_{\theta} / r-\frac{1}{R e} \frac{2 i m}{r^{2}} & \Lambda_{m, k}-\frac{1}{R e} \Delta_{m, r \theta x}^{*} & 0 & \frac{i m}{r} \\
\frac{\partial U_{x}}{\partial r} & 0 & \Lambda_{m, k}-\frac{1}{R e} \Delta_{m, r \theta x} & i k \\
\frac{1}{r}+\frac{\partial}{\partial r} & \frac{i m}{r} & i k & 0
\end{array}\right], \\
\mathcal{B}=\left[\begin{array}{cccc}
i & 0 & 0 & 0 \\
0 & i & 0 & 0 \\
0 & 0 & i & 0 \\
0 & 0 & 0 & 0
\end{array}\right],
\end{gathered}
$$

with $\Delta_{m, r \theta x}=\left(\frac{1}{r} \frac{\partial}{\partial r}+\frac{\partial^{2}}{\partial r^{2}}-k^{2}-\frac{m^{2}}{r^{2}}\right)$ and $\Delta_{m, r \theta x}^{*}=\Delta_{m, r \theta}-\frac{1}{r^{2}}$.

On the other hand, the spatial stability analysis allows the investigation of the spatial evolution of the helical disturbances (Schmid \& Henningson 2012) and the frequency $\omega$ is a real number whereas the wavenumber $k$ is complex. In particular, its real part, $k_{r}$, corresponds to the axial wavenumber and its imaginary part, $-k_{i}$, is the spatial growth rate. Therefore equations (A 1) can be recast as the quadratic eigenvalue problem (2.4) where the linear operators are defined as follows:

$$
\begin{aligned}
& \mathcal{C}_{0}=\left[\begin{array}{cccc}
i m U_{\theta} / r-\frac{1}{R e} \Delta_{m, r \theta}^{*}-i \omega & -2 U_{\theta} / r+\frac{1}{R e} \frac{2 i m}{r^{2}} & 0 & \frac{\partial}{\partial r} \\
\frac{\partial U_{\theta}}{\partial r}+U_{\theta} / r-\frac{1}{R e} \frac{2 i m}{r^{2}} & i m U_{\theta} / r-\frac{1}{R e} \Delta_{m, r \theta}^{*}-i \omega & 0 & \frac{i m}{r} \\
\frac{\partial U_{x}}{\partial r} & 0 & i m U_{\theta} / r-\frac{1}{R e} \Delta_{m, r \theta}-i \omega & 0 \\
\frac{1}{r}+\frac{\partial}{\partial r} & \frac{i m}{r} & 0 & 0
\end{array}\right] \\
& \mathcal{C}_{1}=\left[\begin{array}{cccc}
i U_{x} & 0 & 0 & 0 \\
0 & i U_{x} & 0 & 0 \\
0 & 0 & i U_{x} & i \\
0 & 0 & i & 0
\end{array}\right], \mathcal{C}_{2}=\left[\begin{array}{cccc}
\frac{1}{R e} & 0 & 0 & 0 \\
0 & \frac{1}{R e} & 0 & 0 \\
0 & 0 & \frac{1}{R e} & 0 \\
0 & 0 & 0 & 0
\end{array}\right]
\end{aligned}
$$

with $\Delta_{m, r \theta}=\left(\frac{1}{r} \frac{\partial}{\partial r}+\frac{\partial^{2}}{\partial r^{2}}-\frac{m^{2}}{r^{2}}\right)$ and $\Delta_{m, r \theta}^{*}=\Delta_{m, r \theta}-\frac{1}{r^{2}}$.

Integration by parts of the spatial stability problem using the hermitian scalar product (2.6) yields the adjoint spatial stability problem (2.7), where

$$
\begin{gathered}
\mathcal{C}_{0}^{\dagger}=\left[\begin{array}{cccc}
-i m U_{\theta} / r-\frac{1}{R e} \Delta_{m, r \theta}^{*}+i \omega & \frac{\partial U_{\theta}}{\partial r}+U_{\theta} / r+\frac{1}{R e} \frac{2 i m}{r^{2}} & \frac{\partial U_{x}}{\partial r} & 0 \\
-2 U_{\theta} / r-\frac{1}{R e} \frac{2 i m}{r^{2}} & -i m U_{\theta} / r-\frac{1}{R e} \Delta_{m, r \theta}^{*}+i \omega & 0 & \frac{\partial}{\partial r} \\
0 & \frac{i m}{r} & -i m U_{\theta} / r-\frac{1}{R e} \Delta_{m}+i \omega & 0 \\
\frac{1}{r}+\frac{\partial}{\partial r} & 0 & 0
\end{array}\right] \\
\mathcal{C}_{1}^{\dagger}=\left[\begin{array}{cccc}
-i U_{x} & 0 & 0 & 0 \\
0 & -i U_{x} & 0 & 0 \\
0 & 0 & -i U_{x} & i \\
0 & 0 & i & 0
\end{array}\right], \mathcal{C}_{2}^{\dagger}=\mathcal{C}_{2}
\end{gathered}
$$

and that satisfies the same boundary conditions of the direct problem.

\section{Appendix B. Convergence of the results to grid and computational domain}

We show here the numerical independence of the results from the size of the domain and grid refinement. 
a)

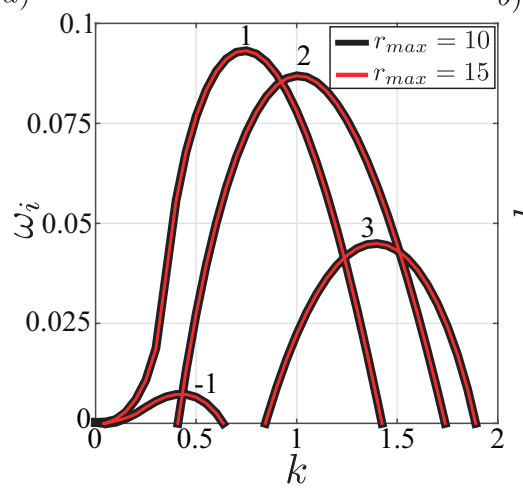

b)

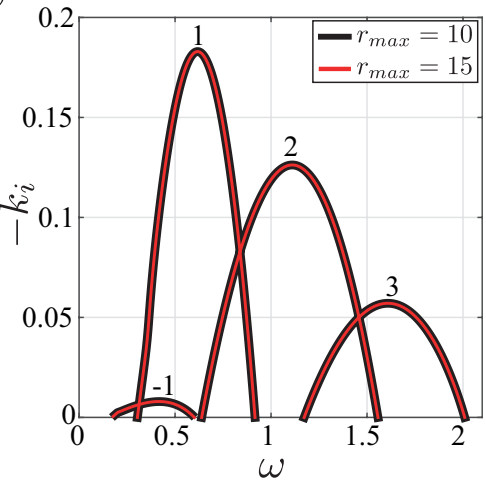

FiguRE 10. a) Temporal and b) spatial growth rates computed with radial extension of the domain equal to $r_{\max }=10$ and $N=40$ (black line) and $r_{\max }=15$ and $N=60$ (red line).
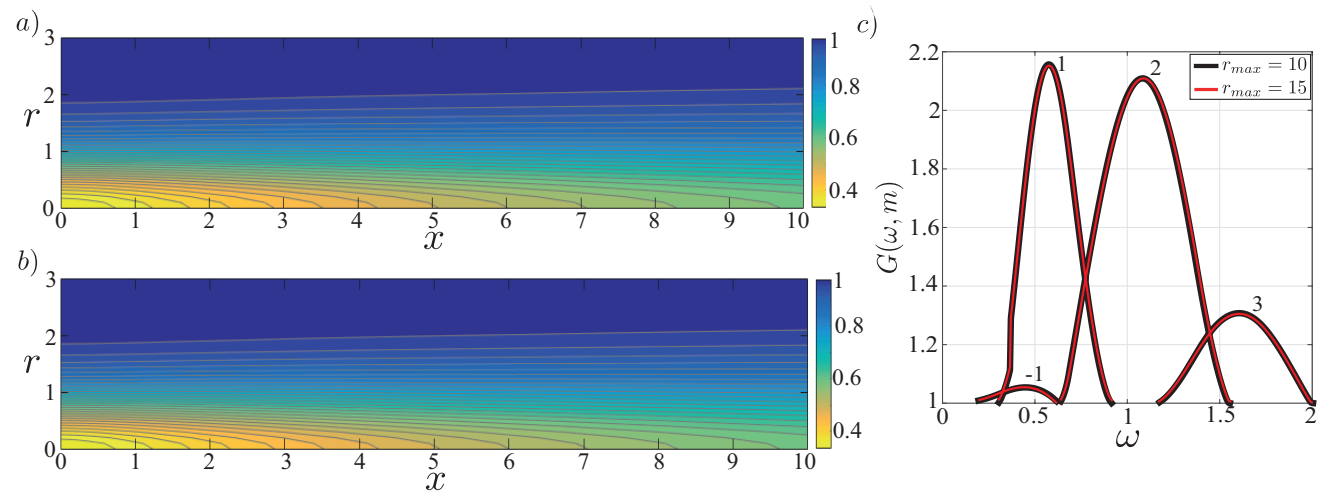

FigURE 11. Streamwise velocity field $U_{x}$ computed using the computational domain a) $\mathcal{D}_{1}$ and b) $\mathcal{D}_{2}$ (see text). The corresponding integral amplification factor $G(\omega, m)$ is shown in c) by black line for $\mathcal{D}_{1}$ and red line for $\mathcal{D}_{2}$

\section{B.1. Local stability analysis}

Figure 10 shows the insensitivity of the (a) temporal and (b) spatial stability analysis of the parallel Batchelor vortex (2.1) on the radial extension of the domain, $r_{\max }$, and on the number of Gauss-Lobatto-Chebyshev (GLC) nodes, $N$. Black line stands for $r_{\max }=$ $10, N=40$ whereas red line indicates $r_{\max }=15, N=60$.

\section{B.2. $D N S$}

Similarly to Figure 3 (c), Figure 11(a) reports the streamwise velocity obtained by using the domain $\mathcal{D}_{1}$ in the DNS, which consists in: $r_{\max }=10$, axial extension of the domain $x_{\max }=20$ and $80 \times 40$ GLC nodes for the velocity components. In (b) the same quantity is obtained by using the larger domain $\mathcal{D}_{2}\left(r_{\max }=15, x_{\max }=25,100 \times 60\right.$ GLC nodes $)$, thus revealing the null influence of the radial and axial extension of the domain and of the free-stress constraint on the base-flow in the region of interest for this study.

\section{B.3. Amplification factor and sensitivity map}

Similar independence on the radial and streamwise extension of the domain is found for the integral amplification factor that is shown in Figure 11(c) by black line in the case of $r_{\max }=10, N=40$ GLC nodes, $N_{x}=30$ and using the $\mathcal{D}_{1}$ base-flow (in the range $x \in[0,10])$. Red line stands for $G(\omega, m)$ computed with $r_{\max }=15, N=60$ GLC nodes, 
$N_{x}=40$ and using the $\mathcal{D}_{2}$ base-flow (for $x \in[0,10]$ ).

In addition, the sensitivity map of the total amplification factor with respect to an inflow base-flow modification is shown in Figure 9(a) by full line when using $r_{\max }=10$, $N=40$ GLC nodes, $N_{x}=30, \mathcal{D}_{1}$ base-flow and $N_{\omega}=210$, and by symbols in the case of $r_{\max }=15, N=60 \mathrm{GLC}$ nodes, $N_{x}=40, \mathcal{D}_{2}$ base-flow and $N_{\omega}=420$. The very good agreement between the sensitivity maps computed with different domain size and grid represents a significant convergence test because all the numerical tools of the analysis are involved: the local spatial analysis, the DNS, integration in $x$ and $\omega$.

\section{REFERENCES}

Åkervik, E., Ehrenstein, U., Gallaire, F. \& Henningson, D. S. 2008 Global twodimensional stability measures of the flat plate boundary-layer flow. European Journal of Mechanics-B/Fluids 27 (5), 501-513.

Batchelor, G.K. 1964 Axial flow in trailing line vortices. Journal of Fluid Mechanics 20 (4), 645-658.

Batchelor, G. K. \& Gill, A. E. 1962 Analysis of the stability of axisymmetric jets. Journal of Fluid Mechanics 14, 529-551.

Bottaro, A., Corbett, P. \& Luchini, P. 2003 The effect of base flow variation on flow stability. Journal of Fluid Mechanics 476, 293-302.

Boujo, E. \& Gallaire, F. 2015 Sensitivity and open-loop control of stochastic response in a noise amplifier flow: the backward-facing step. Journal of Fluid Mechanics 762, 361-392.

Brandt, L., Sipp, D., Pralits, J. O \& Marquet, O. 2011 Effect of base-flow variation in noise amplifiers: the flat-plate boundary layer. Journal of Fluid Mechanics 687, 503-528.

CAmarri, S. 2015 Flow control design inspired by linear stability analysis. Acta Mechanica 226 (4), 979-1010.

CAmarri, S. \& Iollo, A. 2010 Feedback control of the vortex-shedding instability based on sensitivity analysis. Physics of Fluids (1994-present) 22 (9), 094102.

Chомаz, J-M 2005 Global instabilities in spatially developing flows: Non-Normality and Nonlinearity. Annual Review of Fluid Mechanics 37, 357-392.

Crighton, D. G. \& Gaster, M. 1976 Stability of slowly diverging jet flow. Journal of Fluid Mechanics 77, 397-413.

Delbende, I, Chomaz, J-M \& Huerre, P 1998 Absolute/convective instabilities in the Batchelor vortex: a numerical study of the linear impulse response. Journal of Fluid Mechanics 355, 229-254.

Duck, P. W. \& Foster, M. R. 1980 The inviscid stability of a trailing line vortex. Zeitschrift für angewandte Mathematik und Physik ZAMP 31 (4), 524-532.

ECKHOFF, K. S. 1984 A note on the instability of columnar vortices. Journal of Fluid Mechanics 145, 417-421.

FABRE, D \& JACQUin, L 2004 Viscous instabilities in trailing vortices at large swirl numbers. Journal of Fluid Mechanics 500, 239-262.

FABre, D, Sipp, D \& JACQuin, L 2006 Kelvin waves and the singular modes of the lamb-oseen vortex. Journal of Fluid Mechanics 551, 235-274.

Gallaire, F. \& Chomaz, J-M. 2003 Mode selection in swirling jet experiments: a linear stability analysis. Journal of Fluid Mechanics 494, 223-253.

Giannetti, F. \& Luchini, P. 2007 Structural sensitivity of the first instability of the cylinder wake. Journal of Fluid Mechanics 581 (1), 167-197.

HeAton, C. J. 2007 Optimal growth of the Batchelor vortex viscous modes. Journal of Fluid Mechanics 592, 495-505.

Heaton, C. J., Nichols, J. W. \& Schmid, P. J. 2009 Global linear stability of the non-parallel Batchelor vortex. Journal of Fluid Mechanics 629, 139-160.

Huerre, P. \& Rossi, M. 1998 Hydrodynamic instabilities in open flows, chap. 2. Cambridge University Press.

Iungo, G. V., Viola, F., Camarri, S. \& Gallaire, F. 2013 Linear stability analysis of wind 
turbine wakes performed on wind tunnel measurements. Journal of Fluid Mechanics 737, $499-526$.

Khorrami, M. 1991 A Chebyshev spectral collocation method using a staggered grid for the stability of cylindrical flows. International Journal for Numerical Methods in Fluids 12, 825-833.

Leibovich, S. \& Stewartson, K. 1983 Sufficient condition for the instability of columnar vortices. Journal of Fluid Mechanics 126, 335-56.

Lessen, M., Singh, P. J. \& Paillet, F. 1974 The stability of a trailing line vortex. part 1. inviscid theory. Journal of Fluid Mechanics 63 (04), 753-763.

LuDWIEG, H. 1962 Zur erklärung der instabilität der über angestellten deltaflügeln auftretenden freien wirbelkerne. Z. Flugwiss 10 (6), 242-249.

Marquet, O., SipP, D. \& JACQUin, L. 2008 Sensitivity analysis and passive control of cylinder flow. Journal of Fluid Mechanics 615, 221-252.

Mayer, E. W. \& Powell, K. G. 1992 Viscous and inviscid instabilities of a trailing vortex. Journal of Fluid Mechanics 245, 91-114.

Olendraru, C. \& Sellier, A. 2002 Viscous effects in the absolute/convective instability of the Batchelor vortex. J. Fluid Mech. 459, 371-396.

Olendraru, C., Sellier, A., Rossi, M. \& Huerre, P. 1999 Inviscid instability of the Batchelor vortex: Absolute-convective transition and spatial branches. Physics of Fluids 11, 1805-1820.

QAdri, U. A., Mistry, D. \& Juniper, M. P. 2013 Structural sensitivity of spiral vortex breakdown. Journal of Fluid Mechanics 720, 558-581.

Ruith, M. R., Chen, P., Meiburg, E. \& Maxworthy, T. 2003 Three-dimensional vortex breakdown in swirling jets and wakes: direct numerical simulation. Journal of Fluid Mechanics 486, 331-378.

Schmid, P. J. \& Henningson, D. S. 2012 Stability and transition in shear flows, , vol. 142. Springer Science \& Business Media.

Spalart, P. R. 1998 Airplane trailing vortices. Annual Review of Fluid Mechanics 30 (1), $107-138$.

Viola, F., Arratia, C. \& Gallaire, F. 2016 Mode selection in trailing vortices: harmonic response of the non-parallel Batchelor vortex. Journal of Fluid Mechanics 790, 523-552.

Viola, F., Iungo, G.V., Camarri, S., Porté-Agel, F. \& Gallaire, F. 2014 Prediction of the hub vortex instability in a wind turbine wake: stability analysis with eddy-viscosity models calibrated on wind tunnel data. Journal of Fluid Mechanics 750, R1.

Zuccher, S., Bottaro, A. \& Luchini, P. 2006 Algebraic growth in a blasius boundary layer: Nonlinear optimal disturbances. European Journal of Mechanics-B/Fluids 25 (1), 1-17. 